\title{
PAK4 Inhibitor PF-03758309
}

National Cancer Institute

\section{Source}

National Cancer Institute. PAK4 Inhibitor PF-03758309. NCI Thesaurus. Code C84841.

An orally bioavailable small-molecule inhibitor of p21-activated kinase 4 (PAK4) with potential antineoplastic activity. PAK4 inhibitor PF-03758309 binds to PAK4, inhibiting PAK4 activity and cancer cell growth. PAK4, a serine/threonine kinase belonging to the p21-activated kinase (PAK) family, is often upregulated in a variety of cancer cell types and plays an important role in cancer cell motility, proliferation, and survival. 